\title{
Management of white spot and central diastema on anterior teeth with direct composites restoration (a case report)
}

\author{
Adioro Soetojo and Bintang Adiguna Widjaja \\ Departement of Conservative Dentistry, \\ Faculty of Dental Medicine, Universitas Airlangga \\ Surabaya, Indonesia
}

\begin{abstract}
Background: One of the most common aesthetic problems is discoloration and central diastema of the anterior teeth. This can make the patient's appearance disturbed and can lead to confidence in influential patients in the complex interaction of social, cultural and psychological disorders. Purpose: The purpose of this case report is to explain the management of white spot on central incisive with central diastema. Case: A 22 years old woman came to Airlangga Dental Hospital with a chief complaint of shyness and lack of confidence due to some white spot and gap on the two central front teeth. Case Management: The treatment of white spot and central diastema were done using a partial direct veneer technique. Conclusion: White spot and central diastema treated with partial direct veneer restoration is able to produce good aesthetic results.
\end{abstract}

Keywords: diastema central; white spot; direct veneer

Correspondence: Adioro Soetojo, Department of Conservative Dentistry, Faculty of Dental Medicine, Universitas Airlangga. Jl. Prof. Dr. Moestopo No. 47 Surabaya, 60132, Indonesia. Email: adioros@yahoo.com

\section{INTRODUCTION}

In globally, dental caries is one of the most common chronic diseases. It is a multifactorial disease influenced by host, environment, cariogenic bacteria factors that put teeth at increased risk (frequent exposure to dietary carbohydrates and poor oral hygiene). ${ }^{1}$ One of dental caries type is white spot lesion.

White spot lesions are subsurface enamel porosities caused by enamel demineralization. According to Ogaard et al. white spot lesions develop as a result of prolonged plaque accumulation on the enamel surfaces, commonly due to poor oral hygiene. ${ }^{2}$

Some white spot lesions may sometimes remineralize and return to normal or at least to a visually acceptable appearance. However, some white spot lesions persist and become unsightly. In severe cases, white spot lesions may progress to enamel carious lesions which require restorative treatment. Hence, early diagnosis and treatment of these lesions by dentists would be of particular importance. $^{2}$

Various treatments have been proposed to improve the appearance of white spot lesions including restorative procedures, improvement of remineralization using high concentrations of topical fluoride or casein phosphopeptide amorphous calcium phosphate (CPP-ACP), chewing gum to promote remineralization, microabrasion, direct veneer, and argon-laser irradiation. ${ }^{2}$

The spaces between the teeth are called diastema. According to Keen, diastema is defined as the presence of more than $0.5 \mathrm{~mm}$ space between proximal surfaces of adjacent teeth. The presence of diastemas during primary and mixed dentition is natural; these spaces are usually closed by lateral and canine eruption. However, in some cases, diastemas do not close spontaneously, and this may cause aesthetic, psychological and functional disorders. Diastema almost always creates an unpleasant appearance in patients and, depending on the width of the diastema, it affects the speech, especially the pronunciation of the ' $\mathrm{S}$ ' sound. ${ }^{3}$

The etiology of diastemas is multifactorial. In a study in which the frequency of diastemas were investigated; the most common etiological factors; inappropriate fusion of premaxilla (32.9\%) and hypertrophic frenulum (24.4\%) were reported. Microdontia, presence of mesiodens, pegshaped lateral, lateral incisor agenesis, presence of cyst in the midline, dental and skeletal anomalies, muscle deficiencies, finger sucking, tongue thrusting or lip sucking habits, bolton tooth- jaw incompatibility are among the factors that can cause diastema. In addition, periodontitis, trauma, congenital tooth deficiencies, hormonal diseases such as acromegaly can also cause diastemas. Another 
etiological factor of diastemas is genetic. Some researchers have suggested that midline diastemas have autosomal dominant inheritance. Although no specific gene for genetic etiogenesis has been found, there are many syndromes and congenital anomalies that cause midline diastema; Ellis-van Creveld syndrome, like Pai Syndrome etc. ${ }^{3}$

The goal in treating white spot and central diastema is to give back the patient a good smile esthetically. Esthetic restorations always play an important role for emotional and psychological well being of the patient. A beautiful smile quickly radiates excitement, passion, and charisma among the people. ${ }^{1}$

Many various ways can be done to restore central diastema and whitespot on anterior tooth such as direct and indirect composite, and veneers. In modern dentistry, aesthetic anterior composite resin restorations are essential, the common treatment option for these patients is a direct composite resin restoration because of its conservativeness, predictable, repairable and inexpensive. This paper reports how to treat central diastema and white spot on central incisive with direct composites restoration.

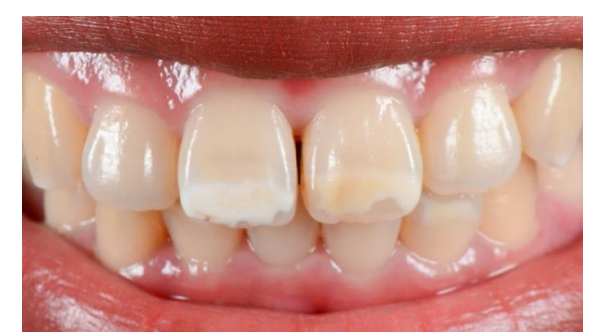

Figure 1. Preoperative look: White spot on tooth 11 and 21 and central diastema.

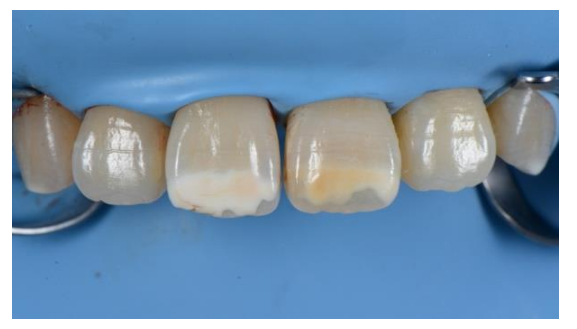

Figure 2. Rubber dam placement to achieve an asepsis area.

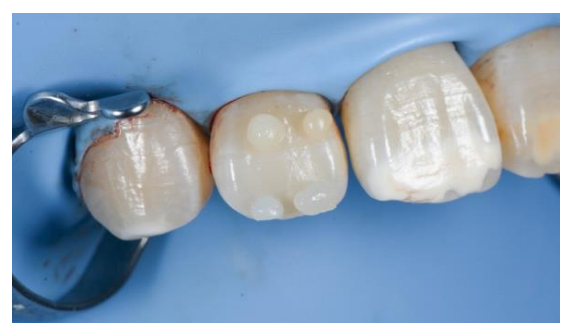

Figure 3. Composite shades taking.

\section{CASE}

A 22 years old woman came to Airlangga Dental Hospital with a chief complaint of shyness and lack of confidence due to some white spot and gap on the two central front teeth. The patient's medical history did not reveal any systemic diseases. The clinical examination showed white spot lesions on tooth 1121 and central diastema between 11 21 (Figures 1). All anterior teeth, as well as the surrounding soft tissues were immaculate and helathy. The decision was made to improve his smile with a conservative, direct adhesive procedure using resin composite.

\section{CASE MANAGEMENT}

All of the treatment were done in one visit, anamnesis, clinical examination, thorough explanation, and initial study to understand about patient's consent in esthetic treatment procedure were done. First, rubber dam was placed to achieve an asepsis area. Blue color sheet was chosen in order to give a contrast color to make it easier in choosing color and doing the multi-layering technique (Figure 2).

After rubber dam was placed, composite shades taking was done by using button shade technique. It was done on lateral incisive. Mass of dentin color was placed on cervical area and mass of enamel color was placed on incisal area then light cured. Micerium UD3 color was chosen for dentin shade and micerium UE2 was chosen for enamel shade (Figure 3). Minimal invasive preparation was done to remove all of the white spot. Bevelling was done to give a better retention of composites and to help blending the color better at the border between the real tooth and the restoration (Figure 4).

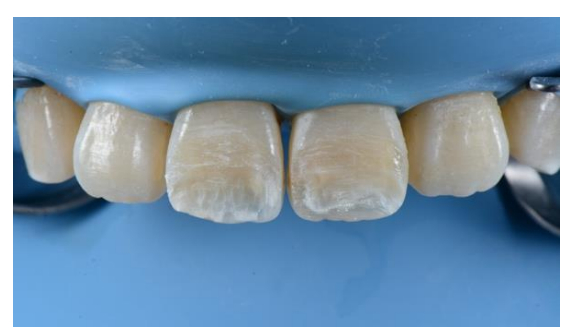

Figure 4. Preparation of the teeth

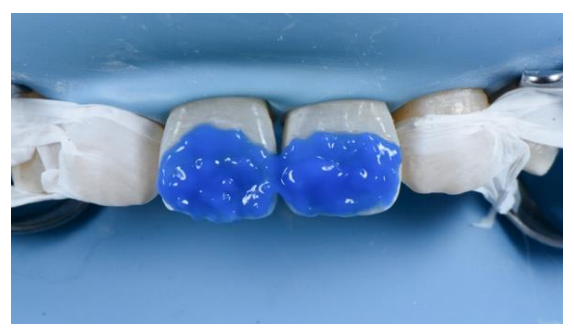

Figure 5. Total etch procedure 
Teflon tape was placed at the lateral incisive to protect lateral teeth from the etch. Total etch technique was done by using $37 \%$ phosphoric acid for 30 s on enamel and 15 s on dentin, then rinsed with water spray and dried (Figure 5).

Thin layer of prime\&bond ${ }^{\circledR}$ one ETCH \& RINSE was applied with rubbing motion on the surface and then dried with compressed air until there was no movement from the bonding and then light cured (Figure 6). Dentin part was layered using UD3 Micerium dentin color. Mamelon was made and then light cured (Figure 7).

Final layering for enamel and diastema closure were done using Micerium UE2 Enamel color then light cured. Sectional matrix were placed vertically to make the proximal contour of the restorations (Figure 8). The finishing was done using abrasive disc and lastly, the restorations were then polished with lowspeed handpiece and Enhance ${ }^{\circledR}$ PoGo ${ }^{\circledR}$ systems (Figure 9 and 10).

\section{DISCUSSION}

Tooth colour is determined by the combination of different optical properties of enamel, dentin and pulp. Extrinsic discolorations can be removed with a prophylactic cleaning

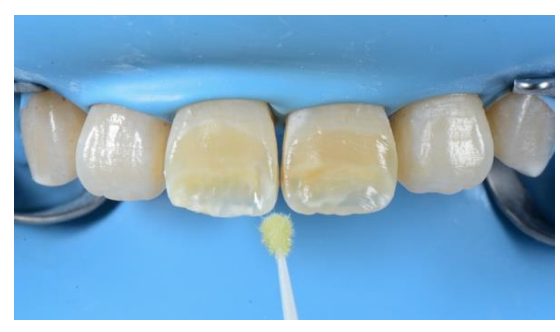

Figure 6. Bonding procedure

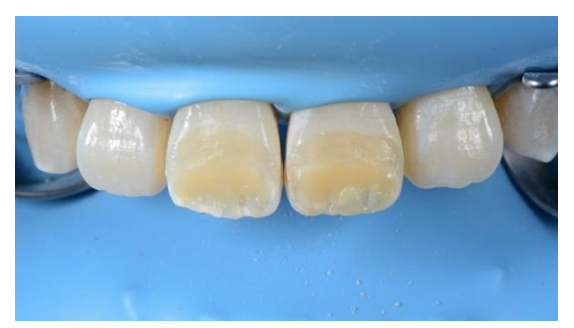

Figure 7. Dentin layering

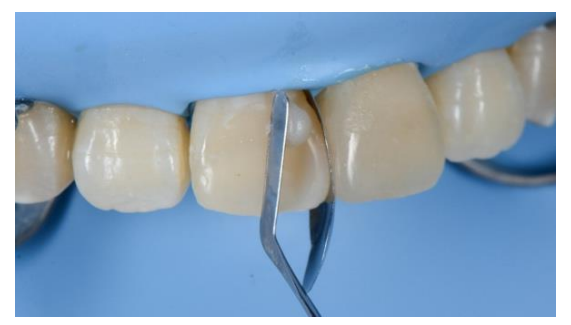

Figure 8. Enamel layering procedure, but intrinsic staining necessitates various treatment options to completely eliminate or even mask the discoloured areas of teeth. ${ }^{1}$

Various methods have been suggested to remove or mask discoloration such as microabrasion, macroabrasion, vital or non-vital bleaching, direct or indirect veneering and full crowns. Conservative treatments like microabrasion, bleaching, and macroabrasion offer advantages over the conventional treatments involving partial or complete coverage restorations. ${ }^{5}$

In this case treatment is done by vital bleaching and direct composite veneer. Vital bleaching is the first step to correct the color of teeth are not done by direct composite veneer (mandible anterior and canine to premolar maxila). Then, the direct composite veneer is to correction the multiple space on second right insisive to second left insisive maxila.

Teeth exhibiting yellow or orange intrinsic discolouration seem to respond best to vital bleaching. Most of the bleaching gels contain $35-40 \%$ hydrogen peroxide because it has a low molecular weight to easily pass through the enamel and dentin. Use of a light to generate heat may accelerate the oxidation reaction of the hydrogen peroxide owing to an immediate whitening of the teeth. This can increase the tooth sensitivity occasionally but that effect is transient. ${ }^{2}$

Composite veneers which require minimal removal of tooth structure by single appointment are one of the best treatment choices for discolored teeth. Although ceramic laminate veneers have some advantages like color stability and high resistance against abrasion, they have also some disadvantages including high cost and long chair side time. ${ }^{5}$ So, vital bleaching and direct full composite veneers were indicated for this case. G-Aenail from GC was used due to its superior mechanical properties and esthetics.

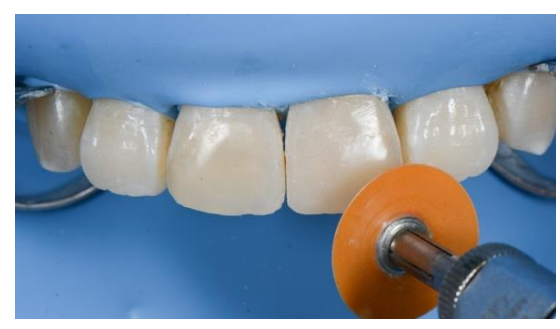

Figure 9. Finishing and polishing

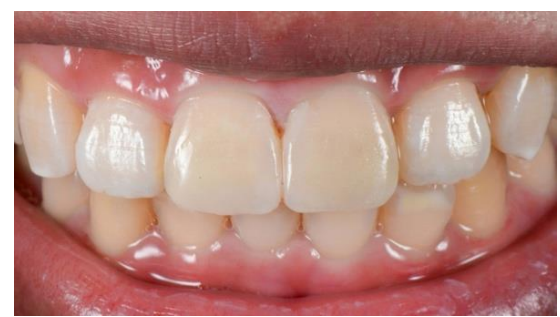

Figure 10. Final result, the restoration color managed to blend naturally and the central diastema already closed. 


\section{CONCLUSION}

A big and deep white lession can be a big concern for some people especially for a young lady. In this case, minimal preparation with bevel to achieve retention and to blend the color well were done before layering the composite to achieve a new shape, diastema closure, and most importantly to change the color of the white spot and make a natural look of the teeth.

Today's composite materials allowed us to perform, fast, long lasting and great looking restoration. New composite properties nowadays had a great handling properties in modeling procedure and excellent aesthetics color especially for anterior restoration, with this composite biomimetism and chameleon effect, the restoration color can blend naturally with the real tooth.

\section{REFERENCES}

1. Ardu S, Krejci I. Biomimetic direct composite stratification technique for the restoration of anterior teeth. Quintessence international. 2006 Mar 1;37(3).

2. Jahanbin, Arezoo, et al. Management of Post-orthodontic White Spot Lesions and Subsequent Enamel Discoloration with Two Microabrasion Techniques. Journal of Dentistry, 2015, 16.1 Suppl: 56.

3. Dindar, Mediha Büyükgöze; Açikgöz, Ece; Atay, Meltem Tekbaş. Closure of The Midline Diastema With Direct Composite Resin Restorations After Frenectomy: Case Report And 1 Year Follow-Up. Journal of Dentistry, 2018, 1.1: 1-4.

4. Wirsching E. Contemporary options for restoration of anterior teeth with composite. Quintessence Int, 2015;46:457-463.

5. Astvaldsdottir A, Dagerhamn J, van Dijken JW, et al. Longevity of posterior resin composite restorations in adults - A systematic review. J Dent, 2015; 43:934-954. 